\title{
Systemic inflammation-based predictors of pathological response to neoadjuvant chemoradiotherapy in locally advanced rectal cancer patients: A propensity score matching analysis
}

\section{Ning Xu}

The first affiliated hospital of Kunming medical university

\section{Wenliang Li ( $\sim$ liwl@ydyy.cn )}

The first affiliated hospital of Kunming medical university

\section{Fengchang Huang}

The affiliated hospital of Kunming medical university

\section{Jun Yang}

The first affiliated hospital of Kunming medical university

\section{Zhengqi Wen}

The first affiliated hospital of Kunming medical university

\section{Liang Yin}

The first affiliated hospital of Kunming medical university

\section{Yunfei Zhang}

The first affiliated hospital of Kunming medical university Jingjiao Zhao

The first affiliated hospital of Kunming medical university

\section{Ruize Zhou}

The first affiliated hospital of Kunming medical university

\section{Jingyu Yang}

The first affiliated hospital of Kunming medical university 
Page $2 / 20$ 


\section{Abstract}

Background: In the management of locally advanced rectal cancer (LARC) treated with neoadjuvant chemoradiotherapy (CRT), the relationship between systemic inflammation-based predictors and tumor response remains unclear. This study aimed to determine whether these inflammatory factors can predict tumor response.

Methods: Totally 205 LARC patients underwent neoadjuvant CRT and curative surgery between 2008 and 2017 were analyzed. After propensity score matching, 146 patients (73 matched pairs) were included in this study. The hematological parameters were collected and their relationship with tumor response was investigated.

Results: After propensity score matching, the neutrophil-lymphocyte-ratio (NLR) and platelet-lymphocyteratio (PLR) before CRT in good response group were significantly lower than those in poor response group, while there was no significant difference in all hematological characteristics between two groups after CRT. The cutoff values of pre-CRT NLR and pre-CRT PLR were 3.10 and 198.7 after receiver operating characteristic analysis. Multivariate analysis model indicated that pre-CRT PLR was not related with tumor response, while pre-CRT NLR $\geq 3.1$ was the predictor of poor tumor response $(\mathrm{OR}=2.047,95 \% \mathrm{Cl}=1.241-3.377$, $p=0.005$ ). Besides, patients with $N L R \geq 3.1$ had a significantly poor tumor regression grade rates compared with patients with NLR<3.1 $(p=0.036)$.

Conclusion: The increased NLR before CRT can be regarded as a hematological factor for poor tumor response in LARC, and higher NLR also represents worse tumor regression grade.

\section{Background}

Colorectal cancer is a common malignant tumor of digestive tract, with high morbidity and mortality[1]. Rectal cancer accounts for about a third of all colorectal cancers[2], of which $45-55 \%$ of patients are diagnosed with locally advanced rectal cancer (LARC) and receive neoadjuvant chemoradiotherapy (CRT)[3]. After CRT, $50-60 \%$ of patients may have different degrees of tumor regression, and $10-30 \%$ of patients may have complete pathological response[4]. For subjects with complete clinical response, conservative operation plan or watch-and-wait strategy is recommended[5]. However, the remaining 50\% non-pathological responders[6], who fail to reduce tumor stage and benefit from CRT, have to bear a heavy financial burden and serious adverse consequences, such as side effects of CRT and tumor progression[7]. Therefore, it is necessary to evaluate the pathological response of neoadjuvant CRT before surgery, thereby improving clinical treatment. Because of the simplicity of blood test, it has good maneuverability for predicting or reflecting the therapeutic effect of CRT.

Tumor-related inflammation has been proved to be a key determinant of disease progression and survival[8]. According to Global Cancer Statistics, chronic inflammation can increase the risk of developing cancer, and is link to more than $15 \%$ of all cancer deaths[9]. Local and systemic inflammatory response is an important prognostic factor for colorectal cancer patients[10]. In recent years, several studies have paid more attention on systemic inflammatory response indicators for predicting the prognosis of rectal cancer after CRT[11-13]. 
In particular, neutrophil-lymphocyte-ratio (NLR), monocyte-lymphocyte-ratio (MLR) and platelet-lymphocyteratio (PLR) are considered as prognostic biomarkers for patients with rectal cancer[13-16]. Some studies have suggested that pre-CRT NLR and PLR, or NLR alone are independently related to tumor response[17, 18]. Ishikawa et al. have suggested that NLR after CRT is more meaningful than that before treatment[12]. Whereas Shen et al. reveled no statistical significance between hematologic parameters and tumor response[19].

Herein, the purpose of this retrospective observational trail was to evaluate the relationship between pathologic tumor response and hematologic parameters before and after CRT in LARC patients via propensity score-matched method, thereby determining whether blood parameters are potential indicators for predicting the tumor pathological response in LARC patients.

\section{Methods}

\section{Patients}

Totally 205 LARC patients from January 2008 to December 2017 were included in this study. Inclusion criteria: the distance to the anal verge was less than $15 \mathrm{~cm}$, and adenocarcinoma was diagnosed by histology. LARC (cT3-4 and / or N+) was evaluated by pelvic high-resolution magnetic resonance imaging, intrarectal ultrasound and CT. All participants were managed with neoadjuvant CRT and total mesorectal excision surgery.

\section{Preoperative chemoradiotherapy and surgery}

All patients underwent intensity modulated radiation therapy (IMRT). The radiation field was the whole pelvis, including the tumor or tumor bed and its surrounding 2-5 cm, presacral lymph nodes and internal iliac lymph nodes. The radiation dose was a total of 45-50.4 Gy with 1.8-2.0 Gy per fractions. These patients received radiotherapy accompanied by oral administration of capecitabine $825 \mathrm{mg} / \mathrm{m}^{2}$ twice a week for 5 weeks. After chemoradiotherapy, they were given 2 cycles of consolidation chemotherapy (oxaliplatin 85 $\mathrm{mg} / \mathrm{m}^{2}$ and capecitabine $1000 \mathrm{mg} / \mathrm{m}^{2}$ ).

Curative surgery was performed 8-11 weeks after the end of chemoradiotherapy, including laparoscopic or open anterior resection (AR) and abdominal-perineal resection (APR) surgery. After surgery, the tumor node metastasis (TNM) staging and pathological tumor regression grading (PTRG) were obtained according to American Joint Committee on Cancer (AJCC) 8th edition TNM staging system[20] and TRG system proposed by Mandard et al.[21]. Besides, all cases were classified into good response (GR) group (ypTNM0-1) and poor response (PR) group (ypTNM2-4) based on pathological TNM (pTNM) classification. The primary endpoint was to evaluate the relationship between hematologic indicators and pathological response.

\section{Hematologic markers}

Hematologic parameters were collected at baseline (pre-CRT) and before surgery (post-CRT). Hemoglobin concentration, white blood cell (WBC) count, neutrophil count, lymphocyte count, monocyte count, platelet 
count, and albumin level were obtained by fully automatic hematology analyzer (Sysmex XE-2100). NLR, MLR and PLR were defined as the ratio of neutrophils, Monocyte and platelets to lymphocytes.

\section{Statistical analysis}

SPSS software (version 23.0, IBM, Armonk, NY, USA) and R (version 3.5) were used for statistical analysis. A propensity score-matched method was adopted via multivariable logistic regression model based on age, gender, body mass index (BMI), distance to the anal verge (DTAV), histologic grade, clinical stage, operation type, hemoglobin and albumin concentration. Paired of patients were derived using 1:1 nearest-neighbor within PS score of 0.03 . This strategy produced 73 matched pairs in each group (Fig. 1).

Continuous variables were described as mean \pm standard deviation for normally distributed variables and median (interquartile range) for abnormally distributed variables. Categorical variables were expressed as absolute numbers (percentage). A Student's t-test or Mann-Whitney U test for continuous variables and Chisquare or Fisher exact test for categorical variables were used to compare the difference between two groups. After matching, both Mcnemar test for categorical variables and pared-samples Wilcoxon rank-sum test for continuous variables were performed. The cutoff point for the continuous variables was determined by the receiver operating characteristic (ROC) curves. Conditional logistic regression was used to define the correlation between the main potential parameters and pathological response of tumors. A two-sides $p$-value $<0.05$ was considered statistically significant.

\section{Results}

\section{Patient characteristics}

There were 105 patients in GR group and 100 patients in PR group. As shown in Table 1, there was significant difference in histologic grade between two groups. After propensity score matching, 73 patients in each group were included in the study, and the difference in histologic grade was eliminated, suggesting that the data were comparable. Besides, the higher pre-CRT and post-CRT carcinoembryonic antigen (CEA) levels were related to the poor tumor response ( $p=0.026$ and 0.002 , respectively).

Table 1 Clinic characteristics of patients 


\begin{tabular}{|c|c|c|c|c|c|c|}
\hline \multirow[t]{2}{*}{ Variables } & \multirow{2}{*}{$\begin{array}{l}\text { All patients } \\
\text { GR group } \\
(n=105)\end{array}$} & \multicolumn{5}{|c|}{ Matched patients } \\
\hline & & $\begin{array}{l}\text { PR group } \\
(n=100)\end{array}$ & $P$ & $\begin{array}{l}\text { GR group } \\
(n=73)\end{array}$ & $\begin{array}{l}\text { PR group } \\
(n=73)\end{array}$ & $P$ \\
\hline Gender, n (\%) & & & 0.436 & & & 0.473 \\
\hline Male & $66(62.9)$ & $69(69.0)$ & & $46(63.0)$ & $51(69.9)$ & \\
\hline Female & $39(37.1)$ & $31(31.8)$ & & $27(37.0)$ & $22(30.1)$ & \\
\hline Age (years), n (\%) & & & 1.000 & & & 0.728 \\
\hline$\geq 60$ & $45(42.9)$ & $43(43.0)$ & & $31(42.5)$ & $34(46.6)$ & \\
\hline$<60$ & $60(57.1)$ & $57(57.0)$ & & $42(57.5)$ & $39(53.4)$ & \\
\hline $\mathrm{BMI}\left(\mathrm{kg} / \mathrm{m}^{2}\right)$ & $22.10 \pm 3.70$ & $22.10 \pm 3.53$ & 0.800 & $22.10 \pm 3.70$ & $22.10 \pm 1.94$ & 0.554 \\
\hline DTAV (cm), n (\%) & & & 0.192 & & & 0.838 \\
\hline$\geq 6$ & $25(23.8)$ & $33(33.0)$ & & $24(32.9)$ & $26(35.6)$ & \\
\hline$<6$ & $80(76.2)$ & $67(67.0)$ & & $49(67.1)$ & $47(64.4)$ & \\
\hline Pre-CRT CEA (ng/ml), r & & & 0.022 & & & 0.026 \\
\hline$\geq 5$ & 33(31.4) & $48(48.0)$ & & $20(27.4)$ & $34(46.6)$ & \\
\hline$<5$ & $72(68.6)$ & $52(52.0)$ & & $53(72.6)$ & $39(53.4)$ & \\
\hline Post-CRT CEA (ng/ml), & (\%) & & 0.005 & & & 0.002 \\
\hline$\geq 5$ & $12(11.4)$ & $28(28.0)$ & & $6(8.2)$ & $21(28.8)$ & \\
\hline$<5$ & $93(88.6)$ & 72(72.0) & & $67(91.8)$ & $52(71.2)$ & \\
\hline Histologic grade, $\mathrm{n}(\%)$ & & & 0.001 & & & 1.000 \\
\hline Low & $1(0.9)$ & 13(13.0) & & $1(1.4)$ & $1(1.4)$ & \\
\hline High & 104(99.1) & $87(87.0)$ & & 72(98.6) & 72(98.6) & \\
\hline Clinical stage, n (\%) & & & 0.345 & & & 1.000 \\
\hline II & $12(11.4)$ & 17(17.0) & & $12(16.4)$ & 13(17.8) & \\
\hline III & 93(88.6) & 83(83.0) & & 61(83.6) & $60(82.2)$ & \\
\hline Operation type, n (\%) & & & 0.196 & & & 1.000 \\
\hline AR & $92(87.6)$ & $80(80.0)$ & & $60(82.2)$ & 61(83.6) & \\
\hline APR & $13(12.4)$ & $20(20.0)$ & & 13(17.8) & $12(16.4)$ & \\
\hline
\end{tabular}


GR: good response; PR: poor response; BMI: Body Mass Index; DTAV: Distance to the anal verge; CRT: chemoradiotherapy; CEA: Carcinoembryonic antigen; AR: Anterior resection; APR: Abdominal-perineal resection.

\section{Hematological characteristics}

Before CRT, there were significant differences in lymphocyte count, NLR, PLR and MLR between GR and PR groups ( $p=0.006,0.002,0.008$ and 0.043 , respectively). After adjusting with propensity score matching, the NLR and PLR in GR group were notably lower than those in PR group ( $p=0.014$ and 0.025 , respectively). No significant difference in lymphocyte count and MLR was noticed between the two groups (Table 2). Moreover, after CRT, there was no statistically significant difference in all hematological characteristics between two groups before and after propensity score matching (Table 3 ).

Table 2 Hematological characteristics before CRT

\begin{tabular}{|lllllll|}
\hline Variables & All patients & & \multicolumn{3}{l|}{ Matched patients } \\
\cline { 2 - 7 } & $\begin{array}{l}\text { GR group } \\
(\mathrm{n}=105)\end{array}$ & $\begin{array}{l}\text { PR group } \\
(\mathrm{n}=100)\end{array}$ & $P$ & $\begin{array}{l}\text { GR group } \\
(\mathrm{n}=73)\end{array}$ & $\begin{array}{l}\text { PR group } \\
(\mathrm{n}=73)\end{array}$ & $P$ \\
\hline $\begin{array}{l}\text { Hemoglobin } \\
(\mathrm{g} / \mathrm{L})\end{array}$ & $145.00 \pm 21.00$ & $138.50 \pm 29.25$ & 0.056 & $140.00 \pm 21.00$ & $141.00 \pm 23.00$ & 0.574 \\
\hline WBC $\left(\times 10^{9} / \mathrm{L}\right)$ & $5.38 \pm 2.34$ & $5.66 \pm 2.24$ & 0.284 & $5.35 \pm 1.36$ & $5.66 \pm 1.94$ & 0.393 \\
\hline $\begin{array}{l}\text { Neutrophil } \\
\left(\times 10^{9} / \mathrm{L}\right)\end{array}$ & $3.28 \pm 1.63$ & $3.42 \pm 1.88$ & 0.265 & $3.24 \pm 1.53$ & $3.41 \pm 1.85$ & 0.433 \\
\hline $\begin{array}{l}\text { Lymphocyte } \\
\left(\times 10^{9} / \mathrm{L}\right)\end{array}$ & $1.58 \pm 0.80$ & $1.27 \pm 0.81$ & 0.006 & $1.58 \pm 0.68$ & $1.43 \pm 0.87$ & 0.149 \\
\hline $\begin{array}{l}\text { Monocyte } \\
\left(\times 10^{9} / \mathrm{L}\right)\end{array}$ & $0.40 \pm 0.22$ & $0.39 \pm 0.19$ & 0.484 & $0.40 \pm 0.21$ & $0.38 \pm 0.18$ & 0.331 \\
\hline $\begin{array}{l}\text { Platelet } \\
\left(\times 10^{9} / \mathrm{L}\right)\end{array}$ & $236.00 \pm 97.00$ & $227.00 \pm 109.00$ & 0.373 & $236.00 \pm 94.00$ & $225.00 \pm 113.00$ & 0.367 \\
\hline Albumin $(\mathrm{g} / \mathrm{L})$ & $42.80 \pm 4.40$ & $42.00 \pm 5.08$ & 0.161 & $42.20 \pm 4.60$ & $42.70 \pm 5.50$ & 0.962 \\
\hline NLR & $2.20 \pm 1.01$ & $2.41 \pm 1.97$ & 0.002 & $2.20 \pm 0.75$ & $2.26 \pm 1.46$ & 0.014 \\
\hline PLR & $146.22 \pm 75.08$ & $174.22 \pm 115.83$ & 0.008 & $141.77 \pm 73.00$ & $159.48 \pm 118.41$ & 0.025 \\
\hline MLR & $0.27 \pm 0.15$ & $0.31 \pm 0.23$ & 0.043 & $0.26 \pm 0.14$ & $0.25 \pm 0.23$ & 0.357 \\
\hline
\end{tabular}

CRT: chemoradiotherapy; GR: good response; PR: poor response; WBC: White blood cell; NLR: neutrophillymphocyte-ratio; MLR: monocyte-lymphocyte-ratio; PLR: platelet-lymphocyte-ratio.

Table 3 Hematological characteristics after CRT 


\begin{tabular}{|c|c|c|c|c|c|c|}
\hline \multirow[t]{2}{*}{ Variables } & \multicolumn{3}{|l|}{ All patients } & \multicolumn{3}{|c|}{ Matched patients } \\
\hline & $\begin{array}{l}\text { GR group } \\
(n=105)\end{array}$ & $\begin{array}{l}\text { PR group } \\
(n=100)\end{array}$ & $P$ & $\begin{array}{l}\text { GR group } \\
(n=73)\end{array}$ & $\begin{array}{l}\text { PR group } \\
(n=73)\end{array}$ & $P$ \\
\hline $\begin{array}{l}\text { Hemoglobin } \\
(\mathrm{g} / \mathrm{L})\end{array}$ & $139.00 \pm 19.00$ & $134.00 \pm 20.00$ & 0.487 & $138.00 \pm 21.00$ & $134.00 \pm 19.00$ & 0.482 \\
\hline $\begin{array}{l}\text { WBC } \\
\left(\times 10^{9} / \mathrm{L}\right)\end{array}$ & $3.68 \pm 1.90$ & $4.20 \pm 2.39$ & 0.076 & $3.68 \pm 1.72$ & $4.18 \pm 2.46$ & 0.113 \\
\hline $\begin{array}{l}\text { Neutrophil } \\
\left(\times 10^{9} / \mathrm{L}\right)\end{array}$ & $2.20 \pm 1.47$ & $2.77 \pm 2.01$ & 0.232 & $2.22 \pm 1.38$ & $2.74 \pm 2.07$ & 0.275 \\
\hline $\begin{array}{l}\text { Lymphocyte } \\
\left(\times 10^{9} / \mathrm{L}\right)\end{array}$ & $0.76 \pm 0.46$ & $0.79 \pm 0.56$ & 0.198 & $0.72 \pm 0.43$ & $0.75 \pm 0.55$ & 0.666 \\
\hline $\begin{array}{l}\text { Monocyte } \\
\left(\times 10^{9} / \mathrm{L}\right)\end{array}$ & $0.40 \pm 0.22$ & $0.44 \pm 0.27$ & 0.611 & $0.38 \pm 0.22$ & $0.46 \pm 0.25$ & 0.093 \\
\hline $\begin{array}{l}\text { Platelet } \\
\left(\times 10^{9} / \mathrm{L}\right)\end{array}$ & $179.00 \pm 77.00$ & $183.50 \pm 71.25$ & 0.226 & $181.00 \pm 64.00$ & $184.00 \pm 55.00$ & 0.678 \\
\hline $\begin{array}{l}\text { Albumin } \\
(\mathrm{g} / \mathrm{L})\end{array}$ & $42.30 \pm 4.80$ & $42.10 \pm 4.05$ & 1.000 & $42.50 \pm 4.80$ & $42.30 \pm 4.30$ & 0.760 \\
\hline NLR & $3.17 \pm 2.32$ & $3.02 \pm 3.12$ & 0.835 & $3.18 \pm 2.42$ & $3.49 \pm 2.82$ & 0.577 \\
\hline PLR & $234.41 \pm 154.82$ & $216.50 \pm 156.02$ & 0.480 & $250.00 \pm 175.50$ & $229.49 \pm 159.58$ & 0.802 \\
\hline MLR & $0.53 \pm 0.37$ & $0.51 \pm 0.43$ & 0.578 & $0.53 \pm 0.35$ & $0.63 \pm 0.40$ & 0.097 \\
\hline
\end{tabular}

CRT: chemoradiotherapy; GR: good response; PR: poor response; WBC: White blood cell; NLR: neutrophillymphocyte-ratio; MLR: monocyte-lymphocyte-ratio; PLR: platelet-lymphocyte-ratio.

\section{Predictive factors for tumor response}

After analyzing the ROC curves of pre-CRT NLR, and pre-CRT PLR (Fig. 2), the cutoff values of pre-CRT NLR and pre-CRT PLR were 3.10 and 198.7, respectively (Table 4), and all patients were divided into two groups based on cutoff values. Factors with significant differences between the two groups were analyzed using a multivariate analysis model (Table 5). The results suggested that post-CRT CEA and pre-CRT NLR were the predictors of tumor response ( $p=0.019$ and 0.005 , respectively), while pre-CRT PLR was not related with tumor response $(p=0.472)$.

Table 4 Predictive value of prognostic factors

\begin{tabular}{|lllll|}
\hline & AUC & $95 \% \mathrm{Cl}$ & sensitivity & specificity \\
\hline pre-CRT NLR & 0.580 & $0.486-0.674$ & 30.1 & 94.5 \\
\hline pre-CRT PLR & 0.578 & $0.485-0.671$ & 34.2 & 82.2 \\
\hline
\end{tabular}


AUC: area under curve; Cl: confidence interval; CRT: chemoradiotherapy; NLR: neutrophil-lymphocyte-ratio; PLR: platelet-lymphocyte-ratio.

Table 5 Multivariate analysis of tumor response

\begin{tabular}{|llll|}
\hline Variables & OR & $95 \% \mathrm{Cl}$ & $P$ \\
\hline Post-CRT CEA $(\geq 5$ vs $<5)$ & 1.839 & $1.107-3.055$ & 0.019 \\
\hline Pre-CRT NLR $(\geq 3.10$ vs $<3.10)$ & 2.047 & $1.241-3.377$ & 0.005 \\
\hline Pre-CRT PLR $(\geq 198.7$ vs $<198.7)$ & 1.356 & $0.591-3.115$ & 0.472 \\
\hline
\end{tabular}

OR: odds ratio; Cl: confidence interval; CRT: chemoradiotherapy; CEA: Carcinoembryonic antigen; NLR: neutrophil-lymphocyte-ratio; PLR: platelet-lymphocyte-ratio.

\section{Survival analysis}

Then, the patients were followed up until they died or until December 31, 2019, of which 59 patients were lost. The median follow-up time was 41 months (11-132 months). During the follow-up period, 32 patients had cancer-specific deaths, and 40 patients had recurrence or metastasis. Patients were divided into two groups based on the threshold of pre-CRT NLR=3.1 and performed survival analysis. The results showed that the 5-year overall survival (OS) of patients with NLR $\geq 3.10$ and NLR $<3.10$ was $73.4 \%$ and $23.2 \%$, and the 3 year disease free survival (DFS) were $74.1 \%$ and $33.5 \%$, respectively. There were significant differences in OS and DFS between two groups $(p<0.001)$ (Figure 3 ).

At the same time, Univariate analysis was performed and pointed out that the worse TNM stage was a risk factor for OS and DFS (Table 6). Then, the COX proportional hazards model was used for multivariate analysis (Table 7). It was found that poor TNM staging was a related factor of 5-year OS (HR=27.858, $p=0.041$ ), while poor TNM staging and higher pre-CRT CEA were related factors of 3-year DFS (HR=8.165, $p=0.015 ; \mathrm{HR}=7.523, p=0.046$ ). However, the increase pre-CRT NLR was not an independent prognostic factor for OS or DFS.

Table 6 Univariate analysis in relation to overall survival and disease free survival 


\begin{tabular}{|c|c|c|c|c|c|c|}
\hline \multirow[t]{2}{*}{ Variables } & \multicolumn{3}{|c|}{ Overall survival } & \multicolumn{3}{|c|}{ Disease-free survival } \\
\hline & $\mathrm{HR}$ & $95 \% \mathrm{Cl}$ & $P$ & HR & $95 \% \mathrm{Cl}$ & $P$ \\
\hline \multicolumn{7}{|l|}{ Pre-CRT NLR } \\
\hline \multicolumn{7}{|l|}{ Low $(<3.1)$} \\
\hline High ( $(\geq 3.1)$ & 3.000 & $0.606-14.864$ & 0.178 & 3.000 & 0.968-9.302 & 0.057 \\
\hline \multicolumn{7}{|l|}{ Pre-CRT PLR } \\
\hline \multicolumn{7}{|l|}{ Low (<198.7) } \\
\hline High ( $\geq 198.7)$ & 1.667 & $0.398-6.974$ & 0.484 & 2.500 & $0.784-7.971$ & 0.121 \\
\hline \multicolumn{7}{|l|}{ урTNM } \\
\hline \multicolumn{7}{|l|}{ урт0-2 NO } \\
\hline урТ3-4/N+ & 11.000 & $1.420-85.201$ & 0.022 & 5.667 & $1.661-19.336$ & 0.006 \\
\hline \multicolumn{7}{|l|}{ Post-CRT CEA } \\
\hline \multicolumn{7}{|l|}{$<5$} \\
\hline$\geq 5$ & 0.750 & $0.168-3.351$ & 0.706 & 1.000 & $0.323-3.101$ & 1.000 \\
\hline \multicolumn{7}{|l|}{ Pre-CRT CEA } \\
\hline \multicolumn{7}{|l|}{$<5$} \\
\hline$\geq 5$ & 3.000 & $0.812-11.081$ & 0.099 & 3.333 & 0.917-12.112 & 0.067 \\
\hline
\end{tabular}

Table 7 Multivariate analysis in relation to overall survival and disease-free survival 


\begin{tabular}{|c|c|c|c|c|c|c|}
\hline \multirow[t]{2}{*}{ Variables } & \multicolumn{3}{|c|}{ Overall survival } & \multicolumn{3}{|c|}{ Disease-free survival } \\
\hline & HR & $95 \% \mathrm{Cl}$ & $P$ & $\mathrm{HR}$ & $95 \% \mathrm{Cl}$ & $P$ \\
\hline \multicolumn{7}{|l|}{ Pre-CRT NLR } \\
\hline \multicolumn{7}{|l|}{ Low $(<3.1)$} \\
\hline $\operatorname{High}(\geq 3.1)$ & 1.284 & $0.097-16.952$ & 0.850 & 3.264 & $0.662-16.101$ & 0.146 \\
\hline \multicolumn{7}{|l|}{ Pre-CRT PLR } \\
\hline \multicolumn{7}{|l|}{ Low (<198.7) } \\
\hline High ( $\geq 198.7)$ & 0.744 & $0.065-8.516$ & 0.812 & 0.642 & $0.102-4.027$ & 0.636 \\
\hline \multicolumn{7}{|l|}{ урTNM } \\
\hline \multicolumn{7}{|l|}{ урт0-2 NO } \\
\hline урТ3-4/N+ & 27.858 & $1.144-678.403$ & 0.041 & 8.165 & $1.514-44.043$ & 0.015 \\
\hline \multicolumn{7}{|l|}{ Post-CRT CEA } \\
\hline \multicolumn{7}{|l|}{$<5$} \\
\hline$\geq 5$ & 0.227 & $0.020-2.563$ & 0.231 & 0.293 & $0.058-1.488$ & 0.139 \\
\hline \multicolumn{7}{|l|}{ Pre-CRT CEA } \\
\hline \multicolumn{7}{|l|}{$<5$} \\
\hline$\geq 5$ & 12.842 & 0.869-189-849 & 0.063 & 7.523 & $1.034-54.754$ & 0.046 \\
\hline
\end{tabular}

\section{The relationship between NLR and pathologic response}

The NLR data of before, during and after CRT were collected and analyzed. As shown in Figure 4A, compared with the NLR before CRT, the NLR was increased during the CRT, and decreased after CRT. In addition, there was no significant difference in the lowest NLR values between the GR and PR groups ( $p=0.533)$ (Figure 4B).

\section{Discussion}

The immune response of patients has important predictive significance not only in clinical prognosis, but also in the effects of radiotherapy and chemotherapy[22]. Through the interaction of systemic and local inflammatory responses, the degree of leukocyte infiltration in tumor varies; besides, each leukocyte subtype, such as neutrophils, lymphocytes, NK cells, dendritic cells, participates in the formation of tumor microenvironment and is closely related to the invasion and metastasis of cancer[9]. Therefore, the assessment of the degree of inflammation in cancer can be used as a biomarker for clinical prognosis and treatment response. The analysis of circulating leukocyte subsets has become the most convenient method, especially for the analysis of NLR, PLR and MLR. Numerous studies have confirmed that they link to the 
prognosis of different cancers such as gastric cancer, ovarian cancer and colorectal cancer [15, 16, 23-25]. NLR, PLR and MLR are known to be predictors of pathological response in LARC patients. Kim et al. suggested that NLR<2.0 and PLR<133.4 before CRT were associated with better tumor response[26]. Kim et al. claimed that patients with baseline NLR $>3$ had poor tumor response[17], while Krauthamer et al. revealed that NLR $<5$ before CRT was related to better tumor response[18]. In this study, hematologic parameters including NLR, PLR and MLR before and after CRT in LARC patients were analyzed to find the predictors of pathological tumor response, and we also adjusted selection bias by using propensity score-matched method. The initial univariate analysis of all patients showed that NLR, PLR and MLR were statistically significant between two groups before CRT, but MLR was not statistically significant after matching. After multivariate analysis, NLR was the only significant predictor, suggesting that NLR $>3.1$ was associated with poor tumor response, with an odds ratio of 2.047, which was similar to the results of Kim. At the same time, the survival analysis also revealed that the patients with NLR>3.1 had poor OS and DFS, while pre-CRT NLR was not an independent prognostic factor for OS or DFS. Overall, pre-CRT NLR may be a potential marker for predicting the tumor pathological response in LARC patients.

Due to the cytotoxic effects of radiotherapy and chemotherapy, necrotic tumor cells increase antigen recognition, and this process changes the local and systemic inflammatory response[27]. Therefore, it is possible to predict tumor response by NLR, PLR and MLR after CRT. Caputo and Ishikawa have suggested that NLR > 3.80 and 3.85 after CRT are predictors of poor tumor response[12, 28]. However, no relationship between hematological factors and tumor response after CRT was observed in our study, which may be related to the uncontrolled factors that affect the systemic inflammatory response in the case enrollment phase. In addition, 6 cases had abnormally elevated neutrophils due to radiotherapy complications, and 9 cases received granulocyte colony stimulating factor treatment during radiotherapy. Moreover, hidden infections might also be potential causes for the differences in the results of this study.

Several studies suggested that lymphopenia was correlated with poor survival[29, 30]. In this study, only NLR before CRT, not lymphocyte count, was predictive factor for tumor response. As shown in Figure $\mathrm{S} 1$, the absolute lymphocyte count (ALC) decreased during CRT and recovered after CRT, which was consistent with the study of Liu et al.[31], while the NLR had an opposite trend to ALC during CRT. However, there was no difference in the ALC and NLR nadir values between different pathological response groups. Since the hematology parameters during CRT would dynamically change with the accumulation of radiotherapy dose, it was difficult to accurately grasp the true lowest point of the entire dynamic process for this kind of blood data collection at a certain time point. In the study of Liu et al., the blood sampling interval during CRT was 7 $\mathrm{d}$, while the sampling interval in this study was $10 \mathrm{~d}$. Different collection intervals might get different values, and there was no research recommending more convincing observation time points at present, which might be the reason for the difference in our results. In addition, the lack of control over confounding factors in retrospective studies also contributed to the difficulty in replicating the results. Although PSM was used to correct some confounding factors, the results were still affected by drugs or infections during CRT, so prospective studies should be designed to further confirm the results.

There are some limitations in this study. This is a retrospective study without controlling the factors that affect the systemic inflammatory response. Secondly, after propensity matching, the sample size shrinks

Page 12/20 
lead to insufficient evidence strength. Thus, prospective studies with a larger sample size are required for further confirmation in the future.

\section{Conclusion}

The increased NLR before CRT can be used as a hematological factor for poor tumor response, and higher NLR also represents worse TRG. It can be used as a simple tool in the clinical management of patients with LARC to help make a better treatment plan and ultimately improve the prognosis.

\section{Abbreviations}

LARC: Locally advanced rectal cancer

CRT: Chemoradiotherapy

NLR: Neutrophil-lymphocyte-ratio

MLR: Monocyte-lymphocyte-ratio

PLR: Platelet-lymphocyte-ratio

AR: Anterior resection

APR: Abdominal-perineal resection

TNM: Tumor node metastasis

pTRG: Pathological tumor regression grading

AJCC: American Joint Committee on Cancer

GR: Good response

PR: Poor response

pTNM: pathological tumor node metastasis

pre-CRT: Hematologic parameters collected at baseline

post-CRT: Hematologic parameters collected before surgery

WBC: White ball cellcount

BMI: Body mass index

DTAV: Distance to the anal verge

ROC: Receiver operating characteristic 
CEA: Carcinoembryonic antigen

\section{Declarations}

\section{Ethics approval and consent to participate}

This study was approved by the Ethics Committee of the First Affiliated Hospital of Kunming Medical University, and all subjects agreed to participate in our study with written informed consent.

\section{Consent for publication}

Not applicable.

\section{Availability of data and materials}

The datasets used and/or analyzed during the current study are available from the corresponding author on reasonable request.

\section{Competing interests}

The authors declare that they have no competing interests.

\section{Funding}

The authors disclosed receipt of the following financial support for the research, authorship, and/or publication of this article: This work was supported by National Natural Science Foundation of China (grant number 31660312), Yunnan Fundamental Research Projects (grant number 2019FA039) and Leading Medical Talents in Yunnan Province (grant number L-2017001).

\section{Authors' contributions}

XN conceived and designed the study, and wrote the manuscript; LWL provided administrative support and collected patient clinicopathological data, and is corresponding author; HFC and YG performed analysis and interpretation of all data; WZQ and ZYF critically read the manuscript; ZJJ, ZRZ and YJY revised the paper. All authors approved the final version of the article to be published.

\section{Acknowledgements}

Not applicable.

\section{References}

[1] F B, J F, I S, RL S, LA T, A J. Global cancer statistics 2018: GLOBOCAN estimates of incidence and mortality worldwide for 36 cancers in 185 countries. CA Cancer J Clin. 2018;68(6):394-424. 
[2] Berardi R, Maccaroni E, Mantello G, Onofri A, Mandolesi A, Bearzi I, et al. Locally advanced rectal cancer: new findings in anticancer therapy. Colorectal Cancer. 2013;2(6):585-601.

[3] NJ B, M D, S R, D C, D T, R A, et al. A rectal cancer feasibility study with an embedded phase III trial design assessing magnetic resonance tumour regression grade (mrTRG) as a novel biomarker to stratify management by good and poor response to chemoradiotherapy (TRIGGER): study protocol for a randomised controlled trial. Trials. 2017;18(1):394.

[4] J B, F S, MR S, S B, RI S, R P, et al. Magnetic Resonance Tumor Regression Grade and Residual Mucosal Abnormality as Predictors for Pathological Complete Response in Rectal Cancer Postneoadjuvant Chemoradiotherapy. Dis Colon Rectum. 2016;59(10):925-33.

[5] M M, RG B-T, DM L, G L, PJ N, SM E, et al. Wait-and-see policy for clinical complete responders after chemoradiation for rectal cancer. Journal of clinical oncology : official journal of the American Society of Clinical Oncology. 2011;29(35):4633-40.

[6] Smith JJ, Garcia-Aguilar J. Advances and Challenges in Treatment of Locally Advanced Rectal Cancer. J Clin Oncol. 2015;33(16):1797-808.

[7] R S, C D, A J. Colorectal cancer statistics, 2014. CA Cancer J Clin. 2014;64(2):104-17.

[8] GJ G, KA C, CS R, PG H, DC M, SJ C. The systemic inflammation-based neutrophil-lymphocyte ratio: experience in patients with cancer. Crit Rev Oncol Hematol. 2013;88(1):218-30.

[9] Mantovani A, Allavena P, Sica A, Balkwill F. Cancer-related inflammation. Nature. 2008;454(7203):43644.

[10] CS R, JM S, PG H, KA O, DC M. The relationship between the local and systemic inflammatory responses and survival in patients undergoing curative surgery for colon and rectal cancers. Journal of gastrointestinal surgery : official journal of the Society for Surgery of the Alimentary Tract. 2009;13(11):2011-8; discussion 8-9.

[11] DC M. The systemic inflammation-based Glasgow Prognostic Score: a decade of experience in patients with cancer. Cancer Treat Rev. 2013;39(5):534-40.

[12] D I, M N, C T, H K, T T, J H, et al. The Role of Neutrophil-to-lymphocyte Ratio on the Effect of CRT for Patients With Rectal Cancer. In vivo (Athens, Greece). 2020;34(2):863-8.

[13] TG K, W P, DH C, HC P, SH K, YB C, et al. Effect of leukocyte alteration on treatment outcomes following preoperative chemoradiotherapy in patients with rectal cancer. Radiation oncology journal. 2017;35(3):217-26.

[14] IH L, S H, SJ L, BW K, D B, HJ K, et al. Systemic Inflammatory Response After Preoperative Chemoradiotherapy Can Affect Oncologic Outcomes in Locally Advanced Rectal Cancer. Anticancer Res. 2017;37(3):1459-65. 
[15] C K, S S, RM M, K L, S S, S B, et al. Prognostic significance of blood inflammatory biomarkers NLR, PLR, and LMR in cancer-A protocol for systematic review and meta-analysis. Medicine. 2019;98(24):e14834.

[16] WH W, N G, KJ R, AC E, F L, ER S, et al. Predictive Value of Leukocyte- and Platelet-Derived Ratios in Rectal Adenocarcinoma. The Journal of surgical research. 2018;232:275-82.

[17] IY K, SH Y, YW K. Neutrophil-lymphocyte ratio predicts pathologic tumor response and survival after preoperative chemoradiation for rectal cancer. BMC Surg. 2014;14:94.

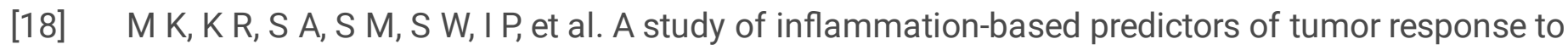
neoadjuvant chemoradiotherapy for locally advanced rectal cancer. Oncology. 2013;85(1):27-32.

[19] $L S, H Z, L L, G L, M ~ F, Y ~ W$, et al. Baseline neutrophil-lymphocyte ratio ( $\geq 2.8)$ as a prognostic factor for patients with locally advanced rectal cancer undergoing neoadjuvant chemoradiation. Radiat Oncol. 2014;9:295.

[20] MR W. AJCC 8th Edition: Colorectal Cancer. Ann Surg Oncol. 2018;25(6):1454-5.

[21] AM M, F D, JC M, J M, M H-A, JF P, et al. Pathologic assessment of tumor regression after preoperative chemoradiotherapy of esophageal carcinoma. Clinicopathologic correlations. Cancer. 1994;73(11):2680-6.

[22] D H, RA W. Hallmarks of cancer: the next generation. Cell. 2011;144(5):646-74.

[23] J Z, HY Z, J L, XY S, CX Z. The elevated NLR, PLR and PLT may predict the prognosis of patients with colorectal cancer: a systematic review and meta-analysis. Oncotarget. 2017;8(40):68837-46.

[24] KL M, AGMT P, WG L. Systematic Review and Meta-Analysis of the Prognostic Significance of Neutrophil-Lymphocyte Ratio (NLR) After R0 Gastrectomy for Cancer. J Gastrointest Cancer. 2018;49(3):23744.

[25] Y Z, S Z, Y L, L Z X S. Prognostic value of systemic inflammatory markers in ovarian Cancer: a PRISMA-compliant meta-analysis and systematic review. BMC Cancer. 2018;18(1):443.

[26] TG K, W P, H K, DH C, HC P, SH K, et al. Baseline neutrophil-lymphocyte ratio and platelet-lymphocyte ratio in rectal cancer patients following neoadjuvant chemoradiotherapy. Tumori. 2019;105(5):434-40.

[27] BA W. Immune checkpoint inhibitors: The linchpins of modern immunotherapy. Immunol Rev. 2019;290(1):6-23.

[28] D C, M C, A C, V LV, M F, R C. Neutrophil to Lymphocyte Ratio (NLR) and Derived Neutrophil to Lymphocyte Ratio (d-NLR) Predict Non-Responders and Postoperative Complications in Patients Undergoing Radical Surgery After Neo-Adjuvant Radio-Chemotherapy for Rectal Adenocarcinoma. Cancer Invest. 2016;34(9):440-51. 
[29] Cho Y, Park S, Byun HK, Lee CG, Cho J, Hong MH, et al. Impact of Treatment-Related Lymphopenia on Immunotherapy for Advanced Non-Small Cell Lung Cancer. Int J Radiat Oncol Biol Phys. 2019;105(5):106573.

[30] Byun HK, Kim N, Yoon HI, Kang SG, Kim SH, Cho J, et al. Clinical predictors of radiation-induced lymphopenia in patients receiving chemoradiation for glioblastoma: clinical usefulness of intensitymodulated radiotherapy in the immuno-oncology era. Radiat Oncol. 2019;14(1):51.

[31] Liu H, Wang H, Wu J, Wang Y, Zhao L, Li G, et al. Lymphocyte nadir predicts tumor response and survival in locally advanced rectal cancer after neoadjuvant chemoradiotherapy: Immunologic relevance. Radiother Oncol. 2019;131:52-9.

\section{Figures}




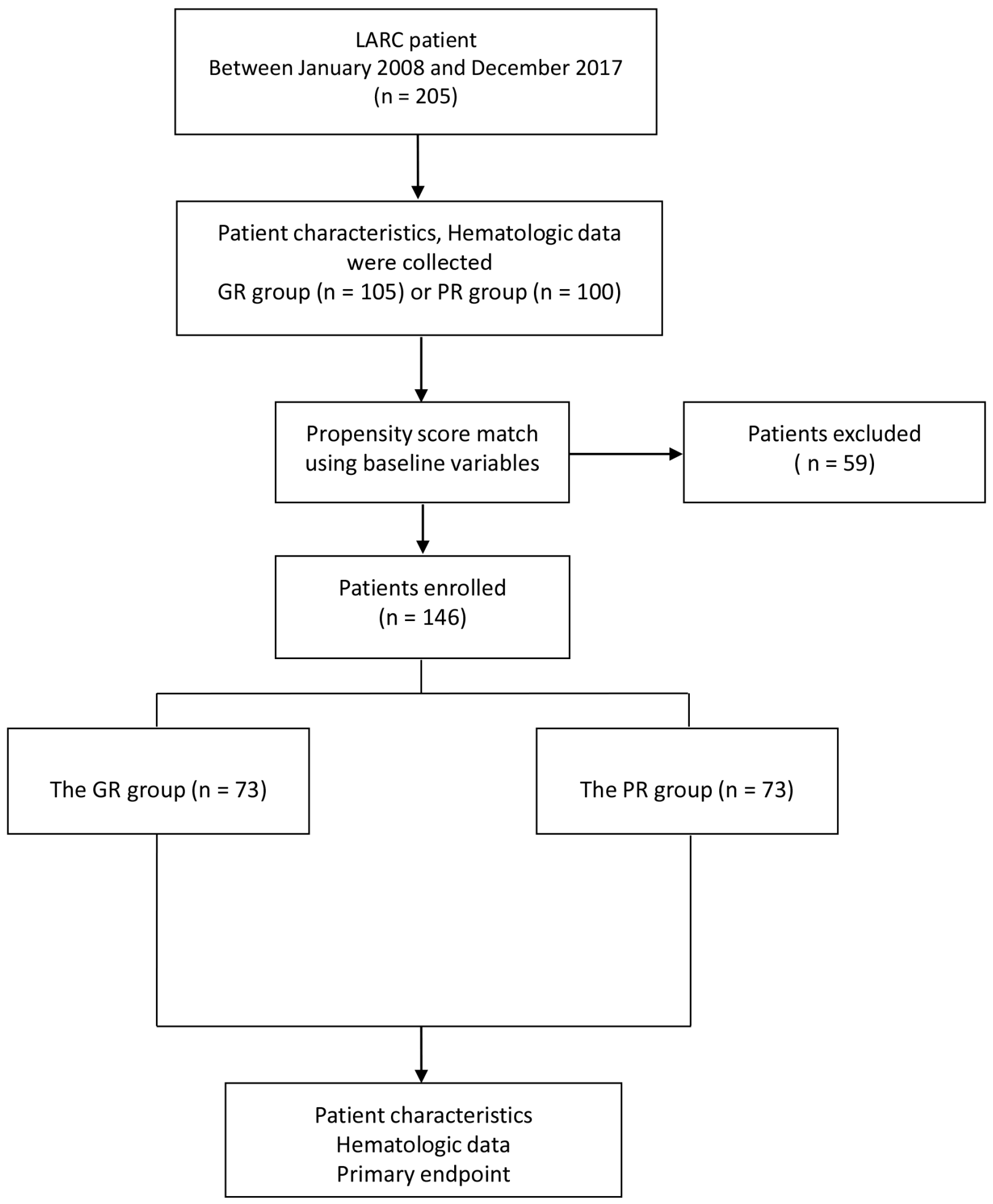

\section{Figure 1}

Flow chart of locally advanced rectal cancer (LARC) patients. 
A

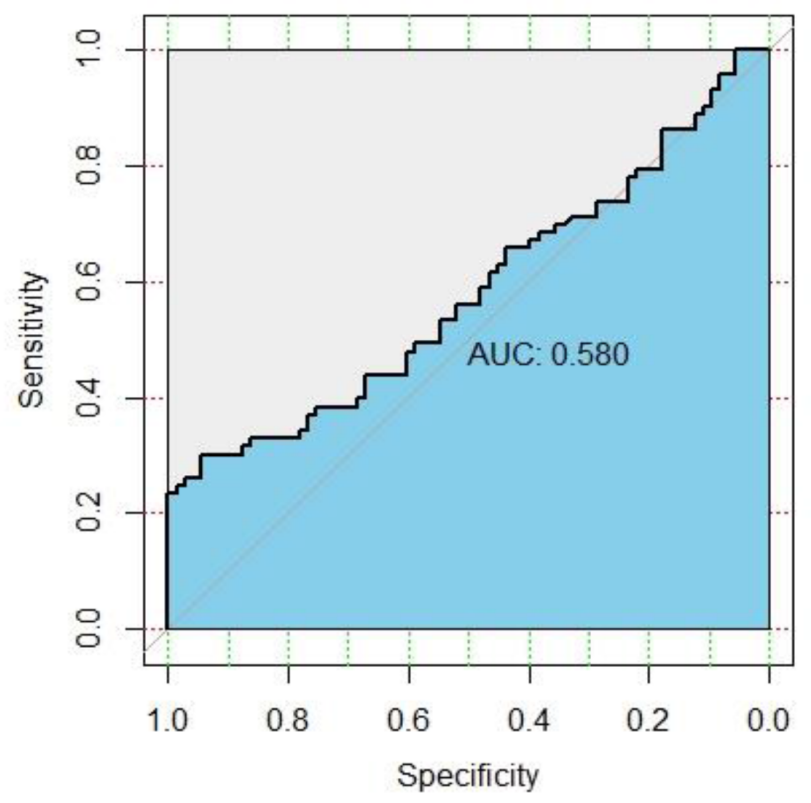

$\mathrm{B}$

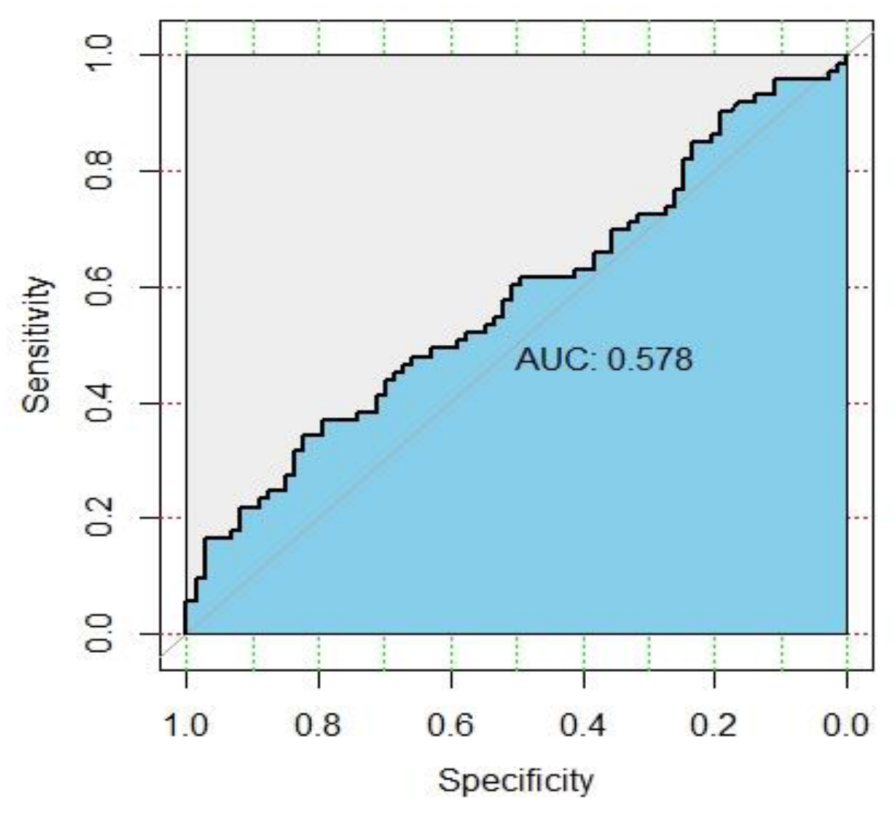

Figure 2

Receiver operating characteristic curves of (A) pre-chemoradiotherapy neutrophil-lymphocyte-ratio (pre-CRT NLR) and (B) pre-chemoradiotherapy platelet-lymphocyte-ratio (pre-CRT PLR) in predicting the tumor response.
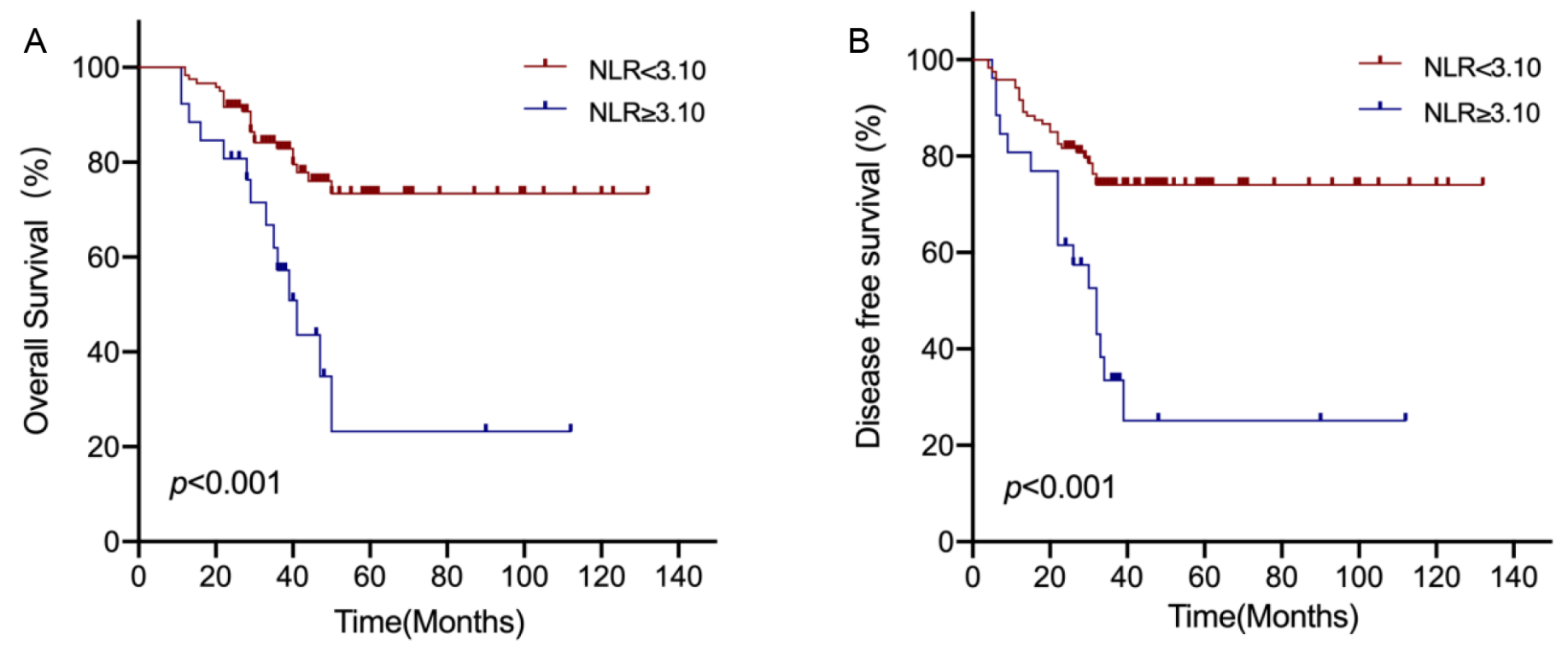

\section{Figure 3}

Kaplan-Meier curves of $(A)$ overall survival and (A) disease free survival of LARC patients with NLR $\geq 3.10$ or NLR $<3.10$. 

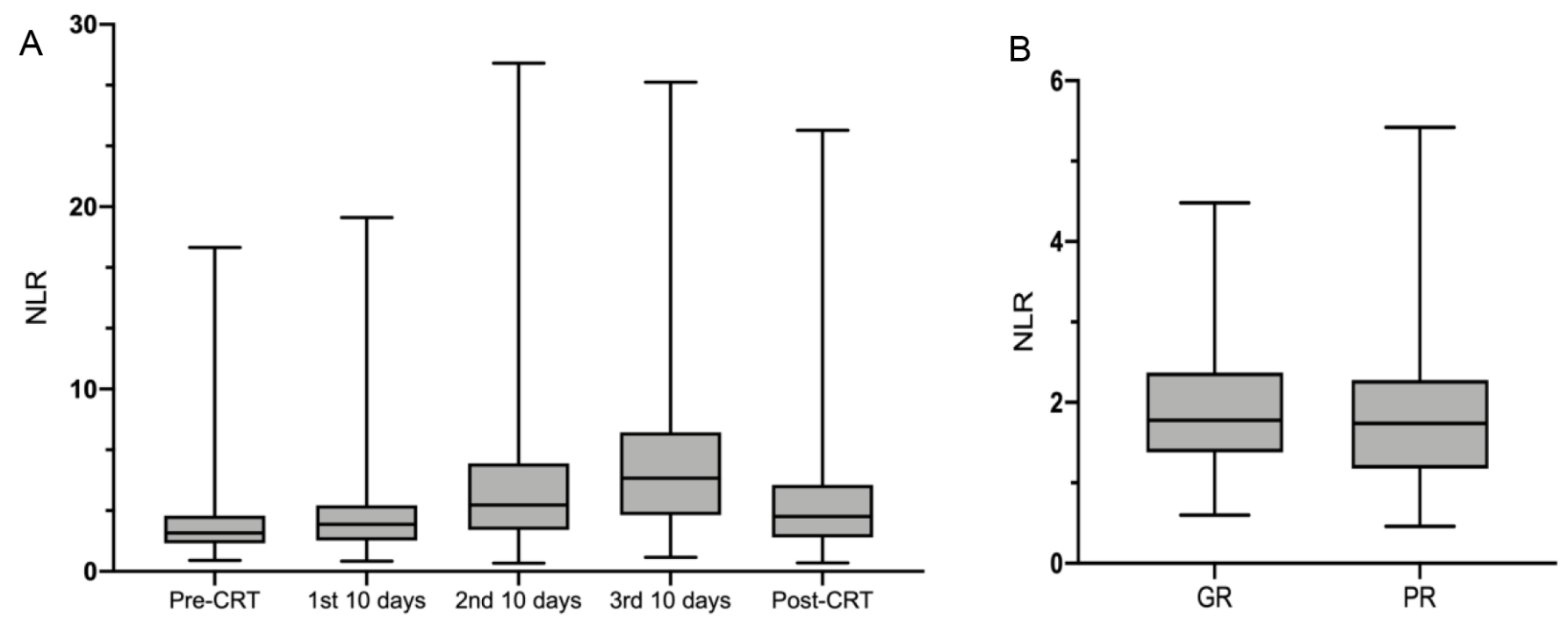

Figure 4

(A) The distribution of NLR values of LARC patients every 10 days during CRT. (B) The NLR nadir values of LARC patients with good response or poor response.

\section{Supplementary Files}

This is a list of supplementary files associated with this preprint. Click to download.

- FigureS1.tif 